\title{
Monte Carlo analysis of noise spectra in self-switching nanodiodes
}

\author{
I. Iñiguez-de-la-Torre, J. Mateos, ${ }^{\text {a) }}$ D. Pardo, and T. González \\ Departamento de Física Aplicada, Universidad de Salamanca, Plaza de la Merced s/n, 37008 Salamanca, \\ Spain
}

(Received 18 October 2007; accepted 20 November 2007; published online 18 January 2008)

\begin{abstract}
By means of a semiclassical two-dimensional Monte Carlo technique, we analyze current noise spectra of InAlAs/InGaAs-based submicron self-switching diodes. Shot noise (at low bias) and diffusion noise in the series resistance (at high bias) are found to dominate the current noise at low frequency (in the plateau beyond the $1 / f$ range). Two peaks of different origins, which may limit the device performance, are found in the noise spectra at higher frequencies. The dependence of the amplitude and frequency of these peaks on the topology of the diodes is analyzed and discussed. Design indications to improve the noise performance of the devices are provided. (C) 2008 American Institute of Physics. [DOI: 10.1063/1.2832505]
\end{abstract}

\section{INTRODUCTION}

Self-switching diodes (SSDs) were originally proposed by Song et al. ${ }^{1}$ By breaking the symmetry of a narrow InGaAs channel (see Fig. 1), this device provides a rectifying behavior (based on surface and electrostatic effects) without the use of any doping junction or barrier structure and can be fabricated with a simple single-step lithographic process. It also exhibits interesting memory properties when operating under appropriate conditions. ${ }^{2,3}$

Since the operation of SSDs is not specifically based on the presence of ballistic transport such as in the case of other III-V nanodevices, ${ }^{4}$ they can also be fabricated with $\mathrm{Si}$ technology, as already done successfully. ${ }^{5}$ However, by using III-V high-mobility material systems (such as those involving InGaAs channels) and downscaling the device dimensions, the operation of SSDs can approach the terahertz range at room temperature, ${ }^{6}$ as experimentally demonstrated in Ref. 7 for an array of these devices working as detector up to $110 \mathrm{GHz}$. At so high frequencies, the intrinsic noise generated by the diodes becomes a performance limitation, and must be carefully analyzed in order to reduce its level as much as possible. To this end, the use of a microscopic technique able to correctly describe nonstationary transport and quasiballistic effects, such as those taking place in these devices, is absolutely necessary. The Monte Carlo (MC) method fulfils these requirements, and it has already been successfully applied to study the static and dynamic behaviors of SSDs. 6,8

The aim of this work is to extend the use of MC simulations to the analysis of noise spectra in InAlAs/ InGaAs-based SSDs. We will identify shot noise in the channel and diffusion noise in the access regions as the main noise sources contributing to the plateau of the current spectral density beyond the $1 / f$ range. At higher frequencies, two peaks are found in the noise spectra, the first one (around $1 \mathrm{THz}$ ) lying in the range of potential applications of SSDs. In order to reduce the noise level in such a frequency inter-

${ }^{a)}$ Electronic mail: javierm@usal.es. val, we will analyze how changes in the geometry of the diodes contribute to modify the noise spectra.

The paper is organized as follows. In Sec. II, details about the simulated structure and the MC model used in the calculations are provided. Section III is devoted to the analysis of the noise spectra, both at low and high frequencies, and their dependence on the device geometry. The main conclusions are drawn in Sec. IV.

\section{PHYSICAL MODEL}

Figure 1 shows the typical topology of SSDs, with their main geometrical parameters: $W_{C}$ channel width, $L$ channel length, $W_{\mathrm{tv}}$ width of the vertical trenches, $W_{\text {th }}$ width of the horizontal trenches, and $L_{\text {acc }}$ length of the accesses. They consist of a narrow semiconductor channel with broken symmetry defined by L-shaped insulating trenches etched on a semiconductor wafer. Thus, just a single-step lithographic process is necessary for their fabrication. The rectifying operation of SSDs is based on the symmetry breaking and the presence of surface states at the sidewalls of the channel. ${ }^{1,6}$

In order to simulate the behaviour of electrons in the device we make use of a semiclassical ensemble MC simulator self-consistently coupled with a two-dimensional (2D)

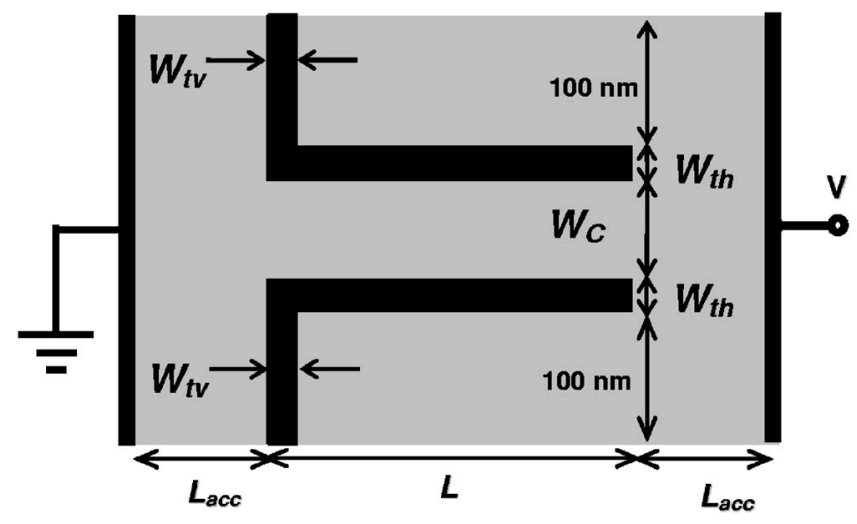

FIG. 1. SSD geometry. $W_{C}$ channel width, $L$ channel length, $W_{\text {tv }}$ and $W_{\text {th }}$ width of the vertical and horizontal trenches, and $L_{\text {acc }}$ length of the accesses to the channel. 


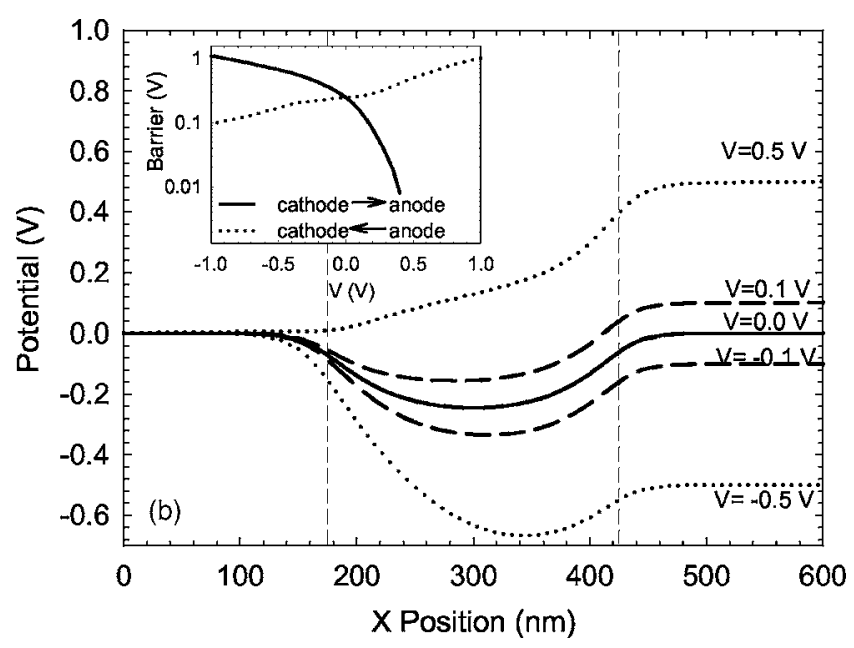

FIG. 2. Profiles of electric potential along the center of the diode for several applied voltages. The vertical lines indicate the limits of the channel. Inset: amplitude of the barriers seen by electrons moving in both directions as a function of the applied voltage.

Poisson solver (PS). The model is essentially the same as that used in Ref. 6 to successfully explain the physics of the $\mathrm{SSD}$ rectifying behavior. It takes into account degeneracy, electron heating, and accurate contact injection with appropriate statistics. The SSDs we are going to analyze are based on the InAlAs/InGaAs/InP layer structure. The simulation is carried out only in the InGaAs channel, where the influence of the fixed positive charges of the whole layer structure is taken into account by assigning a net background doping $N_{\mathrm{Db}}$ of $10^{17} \mathrm{~cm}^{-3}$ when solving Poisson's equation, while the associated impurity scattering is switched off. Details can be found in Refs. 6 and 9. The surface states at the semiconductor-air interfaces originated by the etching process are included in the model by a negative surface charge density $\sigma$ placed at such interfaces. As a novelty, these charges (key in the behavior of SSDs) are updated locally during the simulation according to the surrounding carrier concentration and operating conditions. ${ }^{3,10}$ Simulations are performed at a temperature $T=300 \mathrm{~K}$.

Noise calculations are carried out in the standard way. ${ }^{11}$ First, from the time series of instantaneous current values obtained from the simulation, we evaluate the autocorrelation function of current fluctuations. Then, by Fourier transform, the corresponding spectral density is calculated. To this end, the dynamics of electrons in the device have been simulated during time series of 500000 steps of $1 \mathrm{fs}$ for each bias point.

\section{NOISE RESULTS}

\section{A. Low-frequency noise}

Initially, we will analyze a SSD with a channel length $L=250 \mathrm{~nm}$ and width $W_{C}=50 \mathrm{~nm}$, and trenches of width $W_{\mathrm{tv}}=W_{\mathrm{th}}=5 \mathrm{~nm}$. The length of the accesses is $L_{\mathrm{acc}}=175 \mathrm{~nm}$. The longitudinal profiles of electric potential along the center of the device for several applied voltages are shown in Fig. 2 , where the barrier induced in the channel by the charge at the surface states can be observed. This barrier controls the current flow in both directions. Its amplitude is shown in the

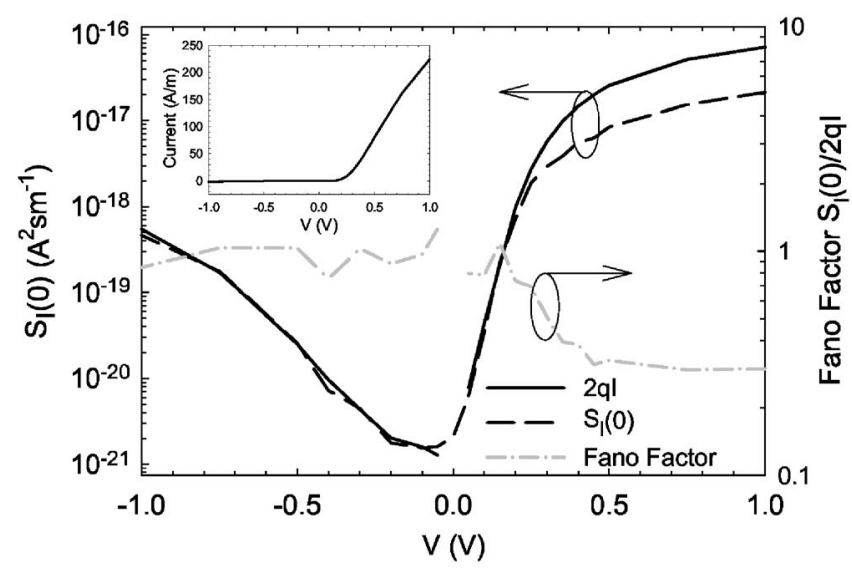

FIG. 3. MC values of $S_{I}(0)$, compared to $2 q I$, as a function of the applied voltage (left axis). The Fano factor $S_{I}(0) / 2 q I$ is also plotted (right axis). The inset shows the $I-V$ curve of the diode in linear scale.

inset. Due to the asymmetry of the diode, the barrier is lowered much more by forward than by reverse applied biases, thus providing the rectifying behavior typical of SSDs, ${ }^{6}$ which is observed in the corresponding $I-V$ curve shown in the inset of Fig. 3. The forward current shows an exponential dependence on the applied voltage for low values of $V$ (as long as the barrier is present), and then becomes linear (resistive behavior), with a tendency to saturation at the highest applied voltages due to hot-carrier effects.

Figure 3 reports the MC values obtained for the spectral density of current fluctuations at low frequency (in the plateau beyond the $1 / f$ range) $S_{I}(0)$, compared to the $2 q I$ value, with $q$ the electron charge. The corresponding Fano factor $F=S_{I}(0) / 2 q I$ is also shown. As long as the barrier limiting the current level has a significant value (higher than about $0.1 \mathrm{eV}$, approximately $4 k_{B} T$ at room temperature, with $k_{B}$ the Boltzmann constant), the SSD displays full shot noise (both under forward and reverse bias), which indicates that transport is barrier controlled and the current is provided by uncorrelated carriers surpassing the barrier. At high forward bias, the barrier is lowered or even disappears (see Fig. 2), the channel resistance decreases, and the diffusive accesses to the channel become more and more important in the total noise of the device. Thus, $S_{I}(0)$, which now essentially corresponds to diffusion noise in the series resistance, shows an increasingly suppressed value with respect to full shot noise. In contrast, in the reverse bias range shown in Fig. 3, the barrier persists and full shot noise is always obtained. This is the typical noise behavior exhibited by SSDs as long as transport in the channel is ballistic or quasiballistic.

We have also calculated the equivalent noise temperature of the diode $T_{n}=S_{I}(0) R / 4 k_{B}$, shown in Fig. 4 as a function of the forward current. $R$ is the low-frequency incremental resistance of the SSD, calculated from the slope of the $I-V$ curve. $T_{n}$ is an important parameter used to experimentally characterize the noise properties of diodes. ${ }^{12,13}$ At low currents, corresponding to the exponential region of the $I-V$ characteristic, the noise temperature is close to half the value of the lattice temperature. This is a feature associated with an ideal exponential dependence of the forward current on the applied voltage $\left[I \propto \exp \left(q V / k_{B} T\right)\right]$, which usually goes along 


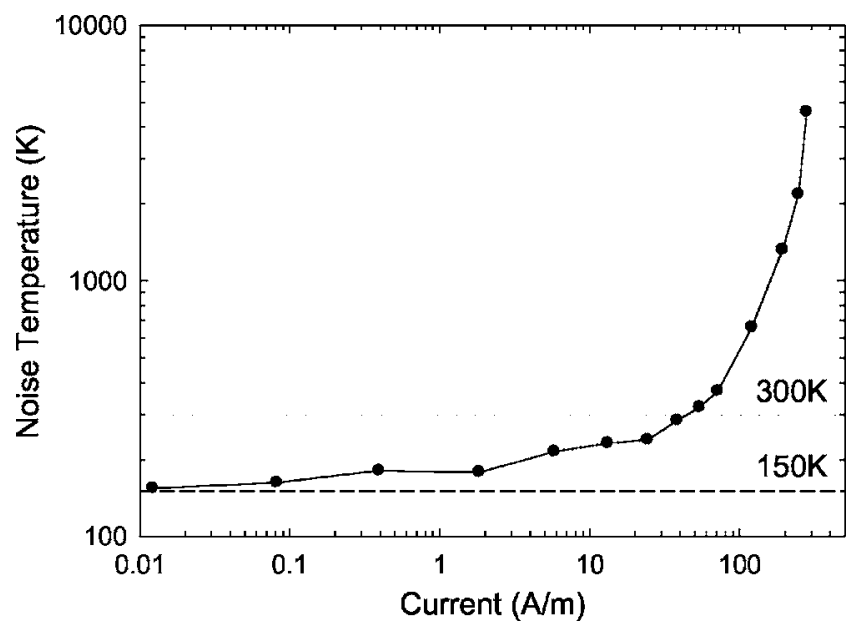

FIG. 4. Noise temperature as a function of the forward current. The horizontal lines indicate room temperature and half its value.

with the previously commented full shot-noise behavior, $S_{I}(0)=2 q I$. As the current increases, the effect of the thermal noise in the series resistance becomes important, since the resistance of the channel decreases exponentially, and the noise temperature increases towards the lattice temperature. Once the barrier disappears (for $V>0.4 \mathrm{~V}, I>50 \mathrm{~A} / \mathrm{m}$ ), a strong electron heating takes place and the noise temperature increases significantly over the lattice temperature. This behavior of the noise temperature is quite similar to that found in other barrier-controlled devices, such as Schottky barrier diodes, ${ }^{12-14}$ except in the very sharp increase observed once the barrier disappears, which is more pronounced in SSDs.

To better understand this increase of the noise temperature related to carrier heating, we plot in Fig. 5 the profiles of electric potential and L valley occupancy along the center of the diode for high forward-bias conditions. For $V=0.4 \mathrm{~V}$, the presence of a small left-to-right barrier is still observed and the $\mathrm{L}$ valley occupancy is practically null all along the device. However, for higher $V$ there is no longer a barrier and the applied voltage is high enough for $\Gamma$ - $L$ intervalley transfer to take place $\left(\Delta E_{\Gamma-\mathrm{L}}=0.45 \mathrm{eV}\right.$ in InGaAs). Due to the quasiballistic motion of electrons in the channel, they hardly

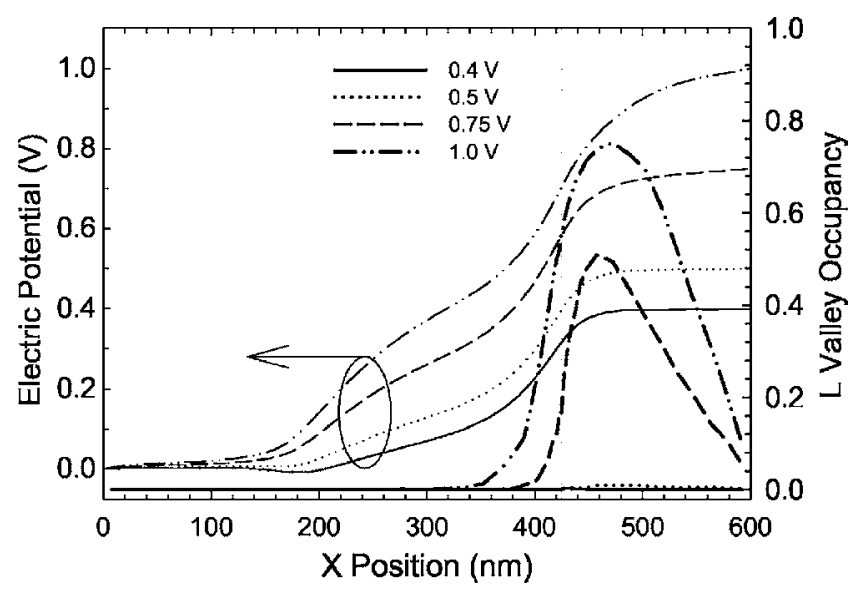

FIG. 5. Profiles of electric potential (left axis) and L valley occupancy (right axis) along the center of the diode for several forward bias conditions. The vertical lines indicate the limits of the channel.

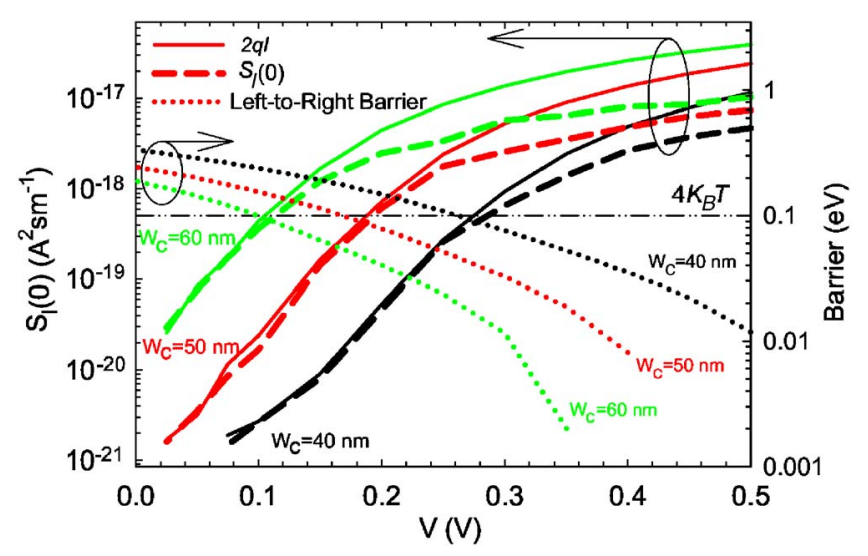

FIG. 6. (Color online) $S_{I}(0)$ compared to $2 q I$ as a function of the applied forward bias for SSDs with different channel widths $W_{C}=40,50$, and $60 \mathrm{~nm}$ (left axis). The corresponding cathode-to-anode barriers are also shown (right axis). The horizontal line indicates the $4 K_{B} T$ value of the barrier.

loose the energy gained from the electric potential, and once this reaches $\Delta E_{\Gamma-\mathrm{L}} / q$ near the end of the channel, a significant presence of $\mathrm{L}$ valley electrons takes place, leading to a much higher differential resistance and, thus, to a sharp increase of $T_{n}$.

To show how the features of $S_{I}(0)$ change when the geometry of the diodes is modified, we have performed simulations in SSDs where the width of the channel $W_{C}$ changes from 40 to $60 \mathrm{~nm}$, keeping the rest of geometrical parameters constant. Figure 6 shows the calculated values of $S_{I}(0)$ compared to $2 q I$ for forward bias conditions. The height of the cathode-to-anode barrier is also shown by the dotted lines. The behavior of $S_{I}(0)$ is essentially the same as already explained for a width of $50 \mathrm{~nm}$ : full shot noise is found while the barrier limiting the current is higher than about $4 K_{B} T$, then diffusion noise becomes important and $S_{I}(0)$ is lower than $2 q I$. What changes from one channel width to another is the bias at which $S_{I}(0)$ departs from $2 q I$. By modifying $W_{C}$, the height of the barrier changes. The closer proximity of the surface charges at both sides of the channel as $W_{C}$ is reduced leads to a larger barrier, so that a higher applied voltage is necessary to reduce its value below $4 K_{B} T$ $\left(V=0.1,0.175\right.$, and $0.26 \mathrm{~V}$, for $W_{C}=60,50$, and $40 \mathrm{~nm}$, respectively).

\section{B. High-frequency noise spectra: Dependence on device topology}

Figure 7 shows the noise spectra $S_{I}(f)$ corresponding to different bias conditions in the diode analyzed in the previous section with $W_{C}$ of $50 \mathrm{~nm}$, which will be the reference structure in our study. In order to identify the influence of the field fluctuations on the total noise, calculations performed when the PS is switched off in the simulations (an average electric field profile previously obtained with the PS on is adopted instead) are also shown for an applied voltage of $0.2 \mathrm{~V}^{14}$

Two main peaks are observed in the spectra. Plasma oscillations are at the origin of the one appearing at the highest frequencies (above $3 \mathrm{THz}$ ). ${ }^{15}$ Stemming from electric field 


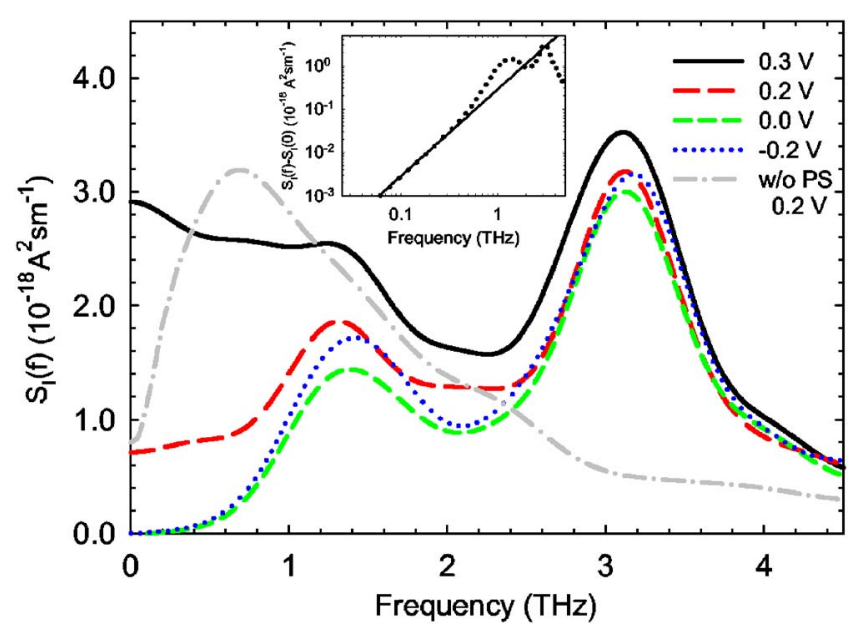

FIG. 7. (Color online) Current noise spectra for several bias conditions in the diode with $W_{C}$ of $50 \mathrm{~nm}$. The case in which the PS is switched off is also shown for $V=0.2 \mathrm{~V}$. The inset illustrates the $f^{2}$ dependence of the noise spectrum for $V=0.0 \mathrm{~V}$.

fluctuations coupled to the carrier movement, plasma oscillations are not present if the PS is switched off, and the associated peak disappears.

The other peak, around $1.3 \mathrm{THz}$, is attributed to returning-carrier effects taking place in the space-charge regions originated by the surface charge at both sides of the vertical trenches. As seen in the inset, which shows the frequency dependence of $S_{I}(f)$ in $\log$ - $\log$ scale for $V=0.0 \mathrm{~V}$, it exhibits the characteristic $f^{2}$ behavior already found in other devices such as Schottky-barrier diodes, ${ }^{12,14}$ revealing a capacitive coupling of the returning-carrier fluctuations to the noise at the terminals. When switching off the PS, this peak does not disappear; it is displaced to lower frequencies and increases in amplitude. We have checked that the frequency of the peak in the spectral density obtained without PS corresponds approximately to the average characteristic time ${ }^{12}$ for carriers injected at the cathode to return to the contact once reflected back in the proximities of the vertical trench, as calculated from MC simulations. As expected, with and without PS, the spectral density takes the same value at low frequency, corresponding to full shot noise associated with the electrons surpassing the barrier and reaching the anode. At high current levels ( $V=0.3 \mathrm{~V}$ in Fig. 7), a significant enhancement of low-frequency noise extending to higher frequencies takes place.

The increase of current noise originated by the peak at lower frequency can be especially problematic for the diode performance, since it could limit the frequency range of potential SSD applications. In the following we will analyze how its amplitude and frequency change with the main parameters of the device topology.

Figure 8 shows noise spectra $S_{I}(f)$ calculated at equilibrium when some parameters of the diode geometry are modified (keeping the others constant): (a) channel width $W_{C},(\mathrm{~b})$ vertical-trench width $W_{\mathrm{tv}}$, (c) channel length $L$, and (d) accesses length $L_{\text {acc }}$. As explained previously, as long as a significant barrier persists (like what happens at equilibrium), the noise at low frequency is essentially determined by the channel, since the barrier limits the current flowing through the diode and the noise associated with the accesses is negligible. In contrast, at high frequency, the regions around the channel have an important weight in the noise. Indeed, as observed in Fig. 8, the level of noise at high frequency is higher the larger is the impedance of the accesses as compared to that of the channel. This explains, for example, why $S_{I}(f)$ is higher when decreasing $L$ or increasing $W_{C}$ or $L_{\text {acc }}$, while it remains with similar amplitude when changing $W_{\mathrm{tv}}$.

Thus, a first possibility to reduce the noise related to the returning-carriers peak is to decrease the resistance of the accesses relative to that of the channel. The best choice to this end is to shorten $L_{\text {acc }}$ [see Fig. 8(d)], which is always desirable to reduce parasitic resistances but may increase the parasitic capacitance between electrodes. Decreasing $W_{C}$ (or increasing $L$ ) would enlarge the channel resistance and reduce the current level, both are undesirable effects. Moreover, a longer channel leads to a poorer high-frequency performance of the device.

A second possibility is to try to move the peak to higher frequencies, thus reducing the amplitude of the noise in the range of interest (below $1 \mathrm{THz}$ ). As observed in Fig. 8, by modifying $W_{C}, L$, or $L_{\text {acc }}$, the frequency of the maximum hardly changes. In contrast, an increase in the width of the vertical trenches $W_{\mathrm{tv}}$ shifts the peak to higher frequencies [Fig. 8(b)]. This is due to the decrease of the capacitance associated with the trenches, which couples the fluctuations originated by the returning carriers at both sides of the trenches to the current at the terminals. However, too wide vertical trenches may deteriorate the rectifying operation of the diode if $W_{\mathrm{tv}}$ becomes comparable to the length of the channel.

Figure 9 shows a simplified equivalent circuit (EC) that allows a qualitative interpretation of the noise spectra in terms of the frequency dependence of the diode impedance $Z(f) ; S_{I}(f)=4 k_{B} T \operatorname{Re}[1 / Z(f)]$ at equilibrium. $R-L-C$ circuits are used in regions where transport is controlled by collisions (accesses to the channel), ${ }^{14,16}$ while the channel, where transport is expected to be ballistic or quasiballistic, is represented by the corresponding $R-C$ circuit. Finally, the geometrical capacitance associated with the vertical trenches $C_{t}=\varepsilon_{0} / W_{\mathrm{tv}}$ is also included, with $\varepsilon_{0}$ the vacuum dielectric constant. The parameters involved in each of the subcircuits of the global EC can be either determined from the diode geometry or estimated from the results of MC simulations. As an example of the type of results obtained from this EC, Fig. 10 shows $\operatorname{Re}[1 / Z(f)]$ when the width of the trenches (and thus the associated capacitance $C_{t}$ ) is modified. As observed, the EC is able to reproduce qualitatively the main features of the noise spectra. Indeed, $\operatorname{Re}[1 / Z(f)]$ exhibits the two peaks observed in $S_{I}(f)$ at similar frequencies. Also, the reduction of $C_{t}$ due to wider vertical trenches leads to a displacement of the first peak to higher frequencies (and, thus, to a decrease of the noise in the range of interest for applications), which supports the interpretation given to $\mathrm{MC}$ results.

\section{CONCLUSIONS}

By means of an ensemble MC simulator selfconsistently coupled with a 2D PS, we have analyzed current 


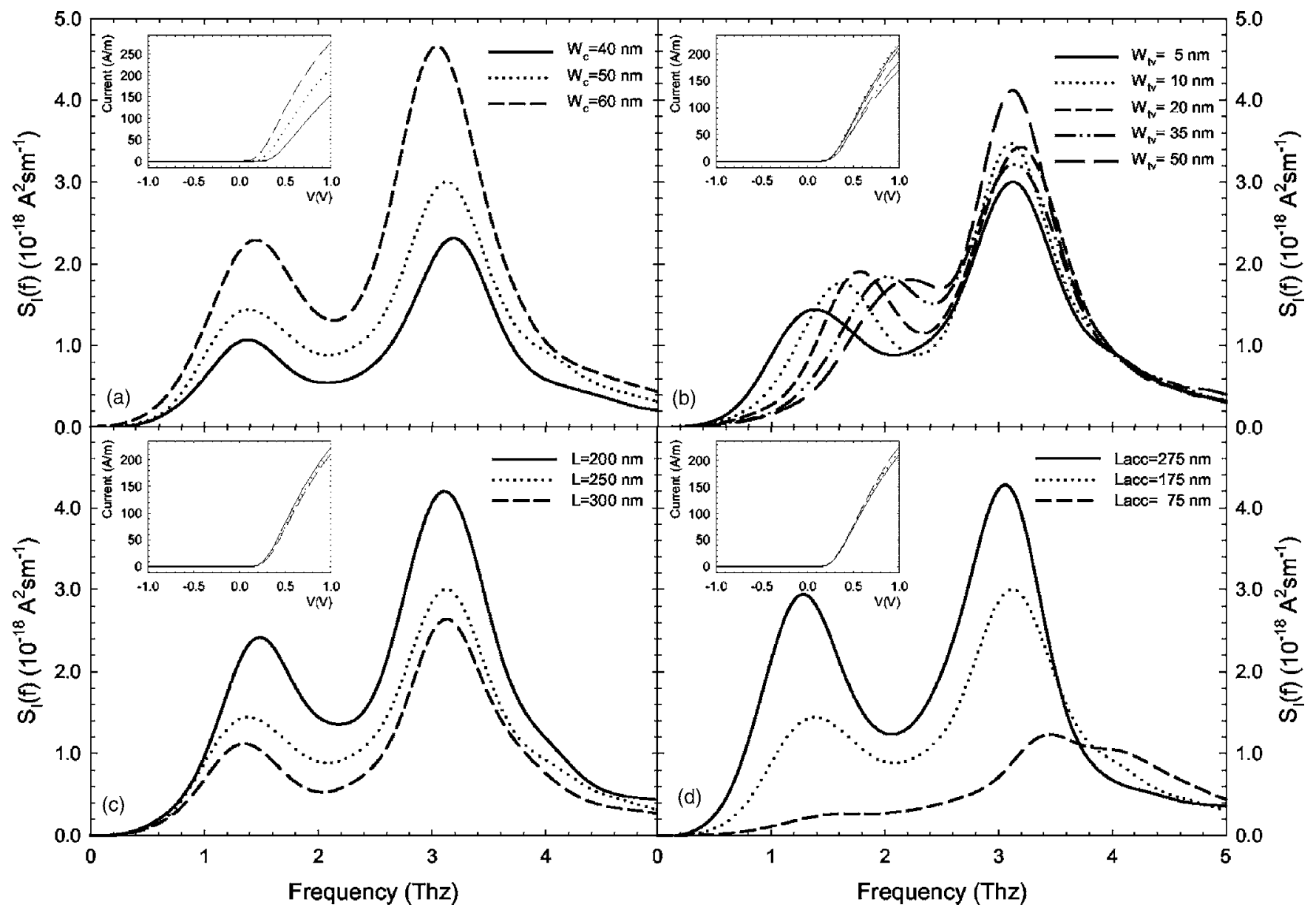

FIG. 8. Current noise spectra at equilibrium $(V=0.0 \mathrm{~V})$ when some parameters of the topology of the diode are modified: (a) channel width $W_{C}=40,50$, $60 \mathrm{~nm}$; (b) width of the vertical trenches $W_{\mathrm{tv}}=5,10,20,35,50 \mathrm{~nm}$; (c) channel length $L=200,250,300 \mathrm{~nm}$; and (d) length of the accesses $L_{\text {acc }}=275$, 175 , $75 \mathrm{~nm}$. The insets show the corresponding $I-V$ characteristics.

noise spectra in SSDs. At low frequency, shot-noise originated by carriers randomly surpassing the barrier present in the channel is found for the lowest currents, when transport is barrier limited. The noise temperature in this range is found to be close to half of the lattice temperature. For higher currents, once the barrier has disappeared, diffusion noise becomes dominant, and the noise temperature reaches and surpasses the lattice temperature due to carrier heating.

At high frequency, two peaks are found in the spectra. The one around $1 \mathrm{THz}$ is especially problematic for potential applications of SSDs. We have studied how to reduce the noise in this range by modifying several parameters of the

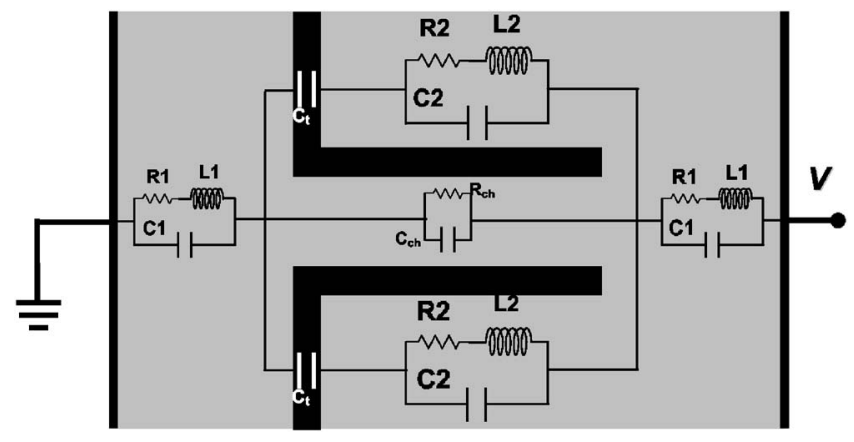

FIG. 9. Equivalent circuit proposed for the SSD. device geometry. The reduction of the accesses and the increase of the width of the vertical trenches have been found to be the best choices for the enhancement of the noise performance, but special care must be taken to avoid deteriorating the rectifying operation and the frequency response of

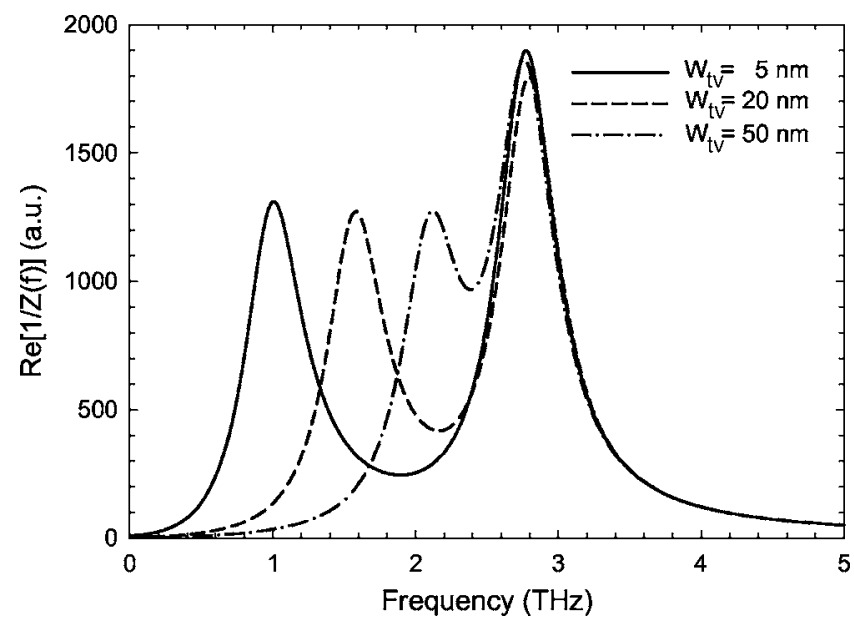

FIG. 10. Real part of the conductance provided by the equivalent circuit of Fig. 9 for several widths of the vertical trenches, $W_{\mathrm{tv}}$, leading to different values of the associated capacitance $C_{t}$. 
SSDs. Finally, an equivalent circuit able to reproduce qualitatively the noise spectra in terms of the device impedance has been proposed.

\section{ACKNOWLEDGMENTS}

This work has been partially supported by the Dirección General de Investigación (MEC, Spain) and FEDER through Project No. TEC2004-05231 and by the Consejería de Educación of the Junta de Castilla y León (Spain) through Project No. SA044A05.

${ }^{1}$ A. M. Song, M. Missous, P. Omling, A. R. Peaker, L. Samuelson, and W. Seifert, Appl. Phys. Lett. 83, 1881 (2003).

${ }^{2}$ A. M. Song, M. Missous, P. Omling, I. Maximov, W. Seifert, and L. Samuelson, Appl. Phys. Lett. 86, 042106 (2005).

${ }^{3}$ I. Iñiguez-de-la-Torre, T. González, D. Pardo, and J. Mateos, Appl. Phys. Lett. 91, 063504 (2007).

${ }^{4}$ L. Worschech, D. Hartmann, S. Reitzenstein, and A. Forchel, J. Phys.: Condens. Matter 17, R775 (2005).

${ }^{5}$ G. Farhi, E. Saracco, J. Beerens, D. Morris, S. A. Charlebois, and J.-P. Raskin, Solid-State Electron. 51, 1245 (2007).
${ }^{6}$ J. Mateos, B. G. Vasallo, D. Pardo, and T. González, Appl. Phys. Lett. 86, $212103(2005)$.

${ }^{7}$ C. Balocco, A. M. Song, M. Aberg, A. Forchel, T. González, J. Mateos, I. Maximov, M. Missous, A. A. Rezazadeh, J. Saijets, L. Samuelson, D. Wallin, K. Williams, L. Worschech, and H. Q. Xu, Nano Lett. 5, 1423 (2005).

${ }^{8}$ K. Y. Xu, G. Wang, and A. M. Song, J. Comput. Electron. 6, 59 (2007). ${ }^{9}$ J. Mateos, B. G. Vasallo, D. Pardo, T. González, J. S. Galloo, Y. Roelens, S. Bollaert, and A. Cappy, Nanotechnology 14, 117 (2003).

${ }^{10}$ I. Iñiguez-de-la-Torre, J. Mateos, T. González, D. Pardo, S. Bollaert, Y. Roelens, and A. Cappy, Semicond. Sci. Technol. 22, 663 (2007).

${ }^{11}$ L. Varani, L. Reggiani, T. Kuhn, T. González, and D. Pardo, IEEE Trans. Electron Devices 88, 11 (1984).

${ }^{12}$ M. Trippe, G. Bosman, and A. van der Ziel, IEEE Trans. Microwave Theory Tech. 34, 1183 (1986).

${ }^{13}$ S. Palczewski, A. Jelenski, A. Grüb, and H. L. Hartnagel, IEEE Microw. Guid. Wave Lett. 2, 442 (1992).

${ }^{14}$ T. González, D. Pardo, L. Reggiani, and L. Varani, J. Appl. Phys. 82, 2349 (1997).

${ }^{15}$ L. Reggiani, P. Golinelli, E. Faucher, L. Varani, T. González, and D. Pardo, Proceedings of the 13th International Conference on Noise in Physical Systems and 1/f Fluctuations, edited by V. Bareikis and R. Katilius (World Scientific, Singapore, 1995), p. 163.

${ }^{16}$ M. Shur, GaAs Devices and Circuits (Plenum, New York, 1987). 